\title{
Statistical Analysis of the Bias in Acoustic Feedback Cancellation System for Hearing Aids
}

\author{
Yasmín Montenegro M. and José C. M. Bermudez
}

\begin{abstract}
The acoustic feedback is one of the main complaints hearing aids users producing distortion of the desired signal and limiting the maximum hearing aid's stable gain. The acoustic feedback cancellation system here presented employs an LMS adaptive estimator and an LMS adaptive predictor operating simultaneously. The nature of the practical problem makes the input to the adaptive estimator and the interference to its output statistically correlated, thus a biased solution is found. This paper presents an analytical model for the bias and the performance of the system is then investigated. Interesting results were obtained from the model. Monte Carlo simulations are presented to verify the accuracy of the derived model.
\end{abstract} aids.

Keywords-Adaptive systems, feedback cancellation, LMS, hearing

\section{INTRODUCTION}

About 660 million people in the world suffer from hearing loss [1]. Several hearing-impaired individuals would benefit from using hearing aids. However, user benefits are still limited due to the lack of systems that can effectively exploit all the potential of signal processing techniques. The desirable hearing aid miniaturization has also created problems. The occlusion effect and the acoustic feedback are among the main user complaints. Venting is essential to reduce the occlusion effect. However, venting leads to undesirable acoustic feedback because of the proximity of microphone and loudspeaker. Acoustic feedback occurs when the hearing aids receiver output signal is fed back into the hearing aids microphone. Large feedforward gains can lead to instability in presence of acoustic feedback. This may lead to signal oscillations which produce annoying sounds referred to as "whistling"and "howling". One way to reduce this problem is to cancel the acoustic feedback using an internal feedback path as shown in Fig. 1, where the acoustic feedback path is modelled by $\boldsymbol{w}^{0}$. Since $\boldsymbol{w}^{0}$ will change in time during normal use [2], [3] (e.g. when the user is giving a hug, chewing or using a telephone), most modern solutions employ adaptive systems. An adaptive filter $\boldsymbol{w}(n)$ is used to generate an estimate $\hat{y}(n)$ of the feedback signal $y(n)$. This estimate is subtracted from the microphone signal. $g(n)$ provides amplification. Ideally, $\hat{y}(n)$ converges to $y(n)$ for $n$ large. Then, only the input signal $x(n)$ is preserved at the hearing aids input $e(n)$ so that $u(n)=g(n) x(n)$.

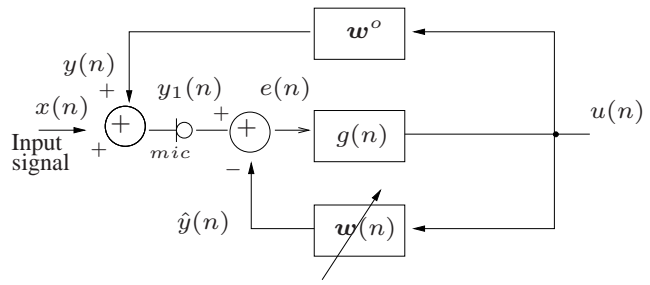

Fig. 1. Basic adaptive feedback cancellation in hearing aids.

Because $g(n)$ is simply a gain, $x(n)$ and $u(n)$ are statistically correlated. Thus, minimum mean-square estimation of $y(n)$ in Fig. 1

Yasmín Montenegro M., University of Antofagasta-Chile and José C. M. Bermudez, Federal University of Santa Catarina-Brazil. E-mails: ymontenegrom@yahoo.com.mx, j.bermudez@ieee.org leads to a biased adaptive weight vector solution. Different schemes have been proposed to reduce this bias. Several of them introduce decorrelating operations such as delays or non-linearities in the forward path $g(n)$ or in the adaptive filter path [4], [5]. The use of restrictions in the adaptive canceller has also been proposed [6][8]. Unfortunately, none of these solutions completely cancels the acoustic feedback.

Alternative solutions have been recently proposed which rely on the direct method of closed-loop identification [9]. The data used for identification are obtained in closed loop but the identification is performed using an open loop model. Recent approaches assume a model for the input signal and apply the prediction-error method (PEM) to reduce the steady-state bias [10]-[13].

In [12] the adaptive filter (called shadow filter) works offline while a fixed cancellation filter operates in the actual signal path. The adaptive filter weights are periodically used to update the cancellation filter response. The adaptive filter updating structure includes an adaptive prediction error filter (PEF) to reduce the solution bias. Both adaptive filters are adapted simultaneously. Though this strucuture has led to good results, no analytical model is available in the literature to predict its behavior and to provide good design guidelines.

In this paper we present a mathematical model for the bias introduced by the adaptive hearing aid feedback cancellation system proposed in [12]. The new model shows the dependence of the adaptive weight bias on the forward path delay and on the adaptation step of the PEF. We present curves that show relationship between the hearing aid signal-to-noise ratio (SNR) and those parameters. Monte Carlo simulations show good agreement with the theoretical predictions.

\section{PROBlem Formulation}

Fig. 2 shows the adaptive feedback cancellation system studied. $x(n)$ is the desired signal and $y(n)$ is the feedback signal to be cancelled. System $\boldsymbol{H}$ and a zero-mean white Gaussian noise $\eta(n)$ define a parametric model for $x(n)$ [12]. $\zeta(n)$ is a zero-mean white Gaussian noise required for identifiability of the feedback path when $x(n)$ includes periodic components [12]. $\boldsymbol{w}^{o}$ is the feedback path to be identified. $\boldsymbol{w}(n)$ is the adaptive estimation filter. $e_{c}(n)$ and $u(n)$ are, respectively, the input and output signals of the hearing aid, which is modeled by a gain $G$ and a delay $D . \boldsymbol{w}^{c}$ is the feedback estimation filter implemented in the signal path. $\boldsymbol{w}^{c}$ is periodically updated with the coefficients of $\boldsymbol{w}(n)$ following some updating policy [12]. The adaptive prediction error filter $\boldsymbol{q}(n)$ whitens $u(n)$ before its use by the adaptive algorithm. The same filter $\boldsymbol{q}(n)$ is also applied to $e(n)$. Note that the top part (above the broken line) of Fig. 2 operates as an stationary system during adaptation of the bottom part $^{1}$. Therefore, signals $u(n)$ and $y_{1}(n)$ are considered stationary during adaptation of $\boldsymbol{w}(n)$ and $\boldsymbol{q}(n)$.

The structure in Fig. 2 is the same proposed in [12] except for the adaptive predictor location. In [12] the adaptive predictor was

${ }^{1}$ We assume a stationary acoustic feedback path in this analysis. Thus, we assume that changes in the acoustic path are slow when compared to the adaptive filter convergence speed. We also assume that transients due to updatings in $\boldsymbol{w}^{c}$ are much shorter than adaptive filter convergence times. 


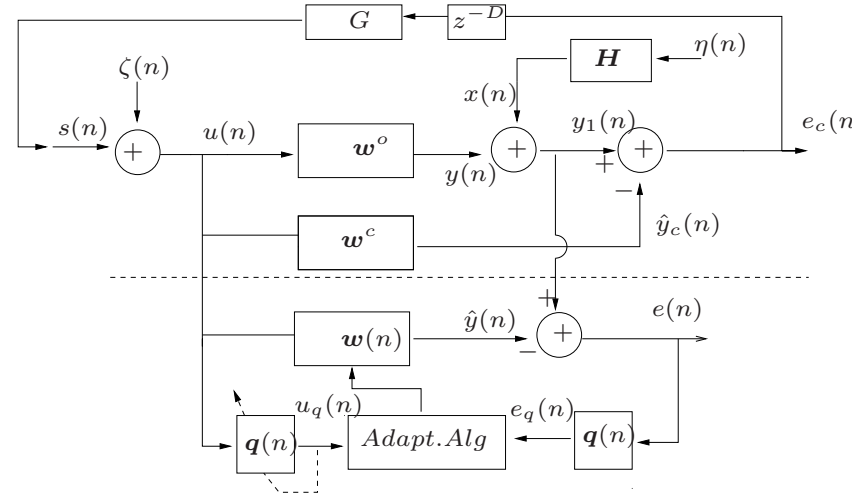

Fig. 2. Hearing aids feedback cancellation structure. Modified from [12].

applied to the error signal $e(n)$ and copied to filter $u(n)$. We propose the structure shown in Fig. 2. This solution pre-whitens the adaptive algorithm input signal and speeds-up convergence of stochastic gradient algorithms [14] whithout affecting the steady-state behavior of the system. We have demonstrated this property in [15].

Under certain conditions, the structure in [12] can provide an unbiased feedback path identification. If $x(n)$ is an autoregressive process, it is shown in [12] that $e(n)$ converges to $x(n)$ and the adaptive PEF (applied to $e(n)$ ) converges to $\boldsymbol{H}^{-1}$. From Fig. 2 and using the subscript $q$ to refer to a signal filtered by $\boldsymbol{q}(n)$,

$$
e_{q}(n)=y_{1_{q}}(n)-\hat{y}_{q}(n) \text {. }
$$

Writing the PEF coefficient vector as $\boldsymbol{q}(n)=\left[1 \boldsymbol{p}^{T}(n)\right]^{T}, \hat{y}_{q}(n)=$ $\hat{y}(n)+\sum_{i=1}^{M} p_{i}(n) \hat{y}(n-i)=\boldsymbol{u}_{q}^{T}(n) \boldsymbol{w}(n)$ with $\boldsymbol{u}_{q}(n)=$ $\left[u_{q}(n), \cdots, u_{q}(N-1)\right]^{T}$, where $N$ is the order of estimator filter, yields

$$
e_{q}(n)=y_{1_{q}}(n)-\boldsymbol{u}_{q}^{T}(n) \boldsymbol{w}(n) .
$$

It is easy to show that $\mathrm{E}\left[e_{q}^{2}(n)\right]$ is minimized for $\boldsymbol{w}(n)=$ $\hat{\boldsymbol{w}}(n)$ where $\hat{\boldsymbol{w}}(n)=\boldsymbol{R}_{u_{q} u_{q}}(n)^{-1} \boldsymbol{r}_{q}(n)$ with $\boldsymbol{R}_{u_{q} u_{q}}(n)=$ $\mathrm{E}\left\{\boldsymbol{u}_{q}(n) \boldsymbol{u}_{q}^{T}(n)\right\}$ and $\boldsymbol{r}_{q}(n)=\mathrm{E}\left\{y_{q 1}(n) \boldsymbol{u}_{q}(n)\right\}$. Using $y_{q 1}(n)=$ $x_{q}(n)+\boldsymbol{u}_{q}^{T}(n) \boldsymbol{w}^{o}$ with $x_{q}(n)=\boldsymbol{q}^{T}(n) \boldsymbol{x}(n)$ we have

$$
\hat{\boldsymbol{w}}(n)=\boldsymbol{R}_{u_{q} u_{q}}(n)^{-1} \mathrm{E}\left\{x_{q}(n) \boldsymbol{u}_{q}(n)\right\}+\boldsymbol{w}^{o}
$$

which shows that the solution bias is controlled by the crosscorrelation vector $\mathrm{E}\left\{x_{q}(n) \boldsymbol{u}_{q}(n)\right\}$ and by the autocorrelation matrix $\boldsymbol{R}_{u_{q} u_{q}}(n)$. Note that (3) has been derived for a fixed PEF (fixed value of $n$ ). In the adaptive scheme, this optimal solution will vary in time as the predictor converges towards its own steady-state solution. In the next section we derive a mathematical model for this bias.

\section{BiAs ANALYSis}

Denoting $\mathcal{B}$ the bias vector, we have from (3) that

$$
\mathcal{B}(n)=\boldsymbol{R}_{u_{q} u_{q}}(n)^{-1} \mathrm{E}\left\{x_{q}(n) \boldsymbol{u}_{q}(n)\right\}
$$

where the time dependence has been explicited. Next we determine mathematical expressions for $\mathrm{E}\left\{x_{q}(n) \boldsymbol{u}_{q}(n)\right\}$ and $\boldsymbol{R}_{u_{q} u_{q}}(n)$.

\section{A. Cross-correlation $E\left\{x_{q}(n) \boldsymbol{u}_{q}(n)\right\}$}

The elements in $\boldsymbol{r}_{x_{q} u_{q}}(n)=\mathrm{E}\left\{x_{q}(n) \boldsymbol{u}_{q}(n)\right\}$ are given by

$$
\begin{aligned}
{\left[\boldsymbol{r}_{x_{q} u_{q}}(n)\right]_{i}=} & \mathrm{E}\left\{x_{q}(n) u_{q}(n-i)\right\} \\
= & \mathrm{E}\left\{\left[x(n)+\boldsymbol{p}^{T}(n) \boldsymbol{x}(n-1)\right]\right. \\
& \left.\times\left[u(n-i)+\boldsymbol{p}^{T}(n) \boldsymbol{u}(n-i-1)\right]\right\}
\end{aligned}
$$

with $i=0, \cdots, N, \boldsymbol{x}(n-1)=[x(n-1), \cdots, x(n-M)]^{T}$ and $\boldsymbol{u}(n-i-1)=[u(n-i-1), \cdots, u(n-i-M)]^{T} . M$ is the order of the predictor. In deriving (5) we assumed slow convergence of the predictor $\boldsymbol{p}(n)$ such that $\boldsymbol{p}(n)=\boldsymbol{p}(n-1)=\boldsymbol{p}(n-N)$.

Define $\boldsymbol{v}_{p}(n)=\boldsymbol{p}(n)-\boldsymbol{p}^{o}$, where $\boldsymbol{p}^{o}$ is the optimum predictor of $x(n)^{2}$ with $\boldsymbol{R}_{u u}(0)=\mathrm{E}\left[\boldsymbol{u}(n) \boldsymbol{u}^{T}(n)\right]$ being the $M \times M$ autocorrelation matrix of $u(n)$ and $r_{u}(l)=E[u(n) u(n-l)]$.

Substituing the value of $\boldsymbol{p}(n)$ in (5) with $\boldsymbol{p}(n)=\boldsymbol{v}_{p}(n)+\boldsymbol{p}^{o}$ we have:

$$
\begin{aligned}
{\left[\boldsymbol{r}_{x_{q} u_{q}}(n)\right]_{i}=} & \mathrm{E}\left\{x(n) u(n-i)+\boldsymbol{v}_{p}^{T}(n) \boldsymbol{u}(n-i-1) x(n)\right. \\
& +\boldsymbol{p}^{o T} \boldsymbol{u}(n-i-1) x(n)+\boldsymbol{v}_{p}^{T}(n) \boldsymbol{x}(n-1) u(n-i) \\
& +\boldsymbol{v}_{p}^{T}(n) \boldsymbol{x}(n-1) \boldsymbol{u}^{T}(n-i-1) \boldsymbol{v}_{p}(n) \\
& +\boldsymbol{v}_{p}^{T}(n) \boldsymbol{x}(n-1) \boldsymbol{u}^{T}(n-i-1) \boldsymbol{p}^{o} \\
& +\boldsymbol{p}^{o T} \boldsymbol{x}(n-1) u(n-i) \\
& +\boldsymbol{p}^{o T} \boldsymbol{x}(n-1) \boldsymbol{u}^{T}(n-i-1) \boldsymbol{v}_{p}(n) \\
& \left.+\boldsymbol{p}^{o T} \boldsymbol{x}(n-1) \boldsymbol{u}^{T}(n-i-1) \boldsymbol{p}^{o}\right\}
\end{aligned}
$$

Conditioning initially the expectation (6) in $\boldsymbol{v}_{p}(n)$ and neglecting the correlations of $\boldsymbol{v}_{p}(n)$ with $u(n)$ and $x(n)$, we have

$$
\begin{aligned}
& {\left[r_{x_{q} u_{q}}(n)\right]_{i}=} \\
& r_{x u}(i)+\left\{\mathrm{E}\left[\boldsymbol{v}_{p}^{T}(n)\right]+\boldsymbol{p}^{o T}\right\} \boldsymbol{r}_{x u}(i+1)+\operatorname{tr}\left\{\boldsymbol{R}_{x u}(i) \boldsymbol{K}_{p}(n)\right\} \\
& +\left\{\mathrm{E}\left[\boldsymbol{v}_{p}^{T}(n)\right]+\boldsymbol{p}^{o T}\right\} \boldsymbol{r}_{u x}(1-i)+\operatorname{tr}\left\{\boldsymbol{R}_{x u}(i) \mathrm{E}\left[\boldsymbol{v}_{p}(n)\right] \boldsymbol{p}^{o T}\right\} \\
& +\operatorname{tr}\left\{\boldsymbol{R}_{x u}(i) \boldsymbol{p}^{o} \mathrm{E}\left[\boldsymbol{v}_{p}^{T}(n)\right]\right\}+\operatorname{tr}\left\{\boldsymbol{R}_{x u}(i) \boldsymbol{p}^{o} \boldsymbol{p}^{o T}\right\}
\end{aligned}
$$

where $\boldsymbol{R}_{x u}(i)=\mathrm{E}[\boldsymbol{x}(n-1) \boldsymbol{u}(n-i-1)], \boldsymbol{r}_{x u}(l-k)=\mathrm{E}[x(n-$ $k) \boldsymbol{u}(n-l)]$ and $\boldsymbol{r}_{u x}(l-k)=\mathrm{E}[u(n-k) \boldsymbol{x}(n-l)]$. The crosscorrelation $r_{x u}(i)$ is derived in the Appendix to be Eq. (37), assuming an autoregressive (AR) model for $x(n)$. Eq. (37) shows that $r_{x u}(i)$ depends on the delay $D$. Thus, $\boldsymbol{r}_{x_{q} u_{q}}(n)$ and hence the bias will change as a function of $D$. A recursion for $\mathrm{E}\left[\boldsymbol{v}_{p}(n)\right]$ using the LMS algorithm has been obtained in [15] as

$$
\mathrm{E}\left[\boldsymbol{v}_{p}(n+1)\right]=\left[\boldsymbol{I}-\rho \boldsymbol{R}_{u u}(0)\right] E\left[\boldsymbol{v}_{p}(n)\right]-\rho\left[r_{u}(1)+\boldsymbol{R}_{u u}(0) \boldsymbol{p}^{o}\right]
$$

where $\rho$ is the predictor step size. In the following we determine a recursion for $\boldsymbol{K}_{p}(n)=\mathrm{E}\left[\boldsymbol{v}_{p}(n) \boldsymbol{v}_{p}^{T}(n)\right]$.

\section{B. Determination of $\boldsymbol{K}_{p}(n)=E\left\{\boldsymbol{v}_{p}(n) \boldsymbol{v}_{p}^{T}(n)\right\}$}

The LMS PEF weight update equation with step size $\rho$ is

$$
\begin{aligned}
\boldsymbol{p}(n+1)= & \boldsymbol{p}(n)-\rho u_{q}(n) \boldsymbol{u}(n-1) \\
= & \boldsymbol{p}(n)-\rho u(n) \boldsymbol{u}(n-1) \\
& -\rho \boldsymbol{u}(n-1) \boldsymbol{u}^{T}(n-1) \boldsymbol{p}(n)
\end{aligned}
$$

Using $\boldsymbol{p}(n)=\boldsymbol{v}_{p}(n)+\boldsymbol{p}^{o}$ in (9) yields

$$
\begin{aligned}
\boldsymbol{v}_{p}(n+1) & =\boldsymbol{v}_{p}(n)-\rho \boldsymbol{u}(n-1) \boldsymbol{u}^{T}(n-1) \boldsymbol{v}_{p}(n) \\
& -\rho \boldsymbol{u}(n-1) \boldsymbol{u}^{T}(n-1) \boldsymbol{p}^{o}-\rho u(n) \boldsymbol{u}(n-1)
\end{aligned}
$$

Multiplying (10) by its transpose,

${ }^{2}$ Note that $\boldsymbol{p}^{o}$ is not necessarily the optimum predictor of $u(n)$ in the mean-square sense 


$$
\begin{aligned}
& \boldsymbol{v}_{p}(n+1) \boldsymbol{v}_{p}^{T}(n+1) \\
& =\boldsymbol{v}_{p}(n) \boldsymbol{v}_{p}^{T}(n)-\rho \boldsymbol{v}_{p}(n) \boldsymbol{v}_{p}^{T}(n) \boldsymbol{u}(n-1) \boldsymbol{u}^{T}(n-1) \\
& -\rho \boldsymbol{v}_{p}(n) \boldsymbol{p}^{o T} \boldsymbol{u}(n-1) \boldsymbol{u}^{T}(n-1)-\rho u(n) \boldsymbol{v}_{p}(n) \boldsymbol{u}^{T}(n-1) \\
& -\rho \boldsymbol{u}(n-1) \boldsymbol{u}^{T}(n-1) \boldsymbol{v}_{p}(n) \boldsymbol{v}_{q}^{T}(n) \\
& +\rho^{2} \boldsymbol{u}(n-1) \boldsymbol{u}^{T}(n-1) \boldsymbol{v}_{p}(n) \boldsymbol{v}_{p}^{T}(n) \boldsymbol{u}(n-1) \boldsymbol{u}^{T}(n-1) \\
& +\rho^{2} \boldsymbol{u}(n-1) \boldsymbol{u}^{T}(n-1) \boldsymbol{v}_{p}(n) \boldsymbol{p}^{o T} \boldsymbol{u}(n-1) \boldsymbol{u}^{T}(n-1) \\
& +\rho^{2} u(n) \boldsymbol{u}(n-1) \boldsymbol{u}^{T}(n-1) \boldsymbol{v}_{p}(n) \boldsymbol{u}^{T}(n-1) \\
& -\rho \boldsymbol{u}(n-1) \boldsymbol{u}^{T}(n-1) \boldsymbol{p}^{o} \boldsymbol{v}_{p}^{T}(n) \\
& +\rho^{2} \boldsymbol{u}(n-1) \boldsymbol{u}^{T}(n-1) \boldsymbol{p}^{o} \boldsymbol{v}_{p}^{T}(n) \boldsymbol{u}(n-1) \boldsymbol{u}^{T}(n-1) \\
& +\rho^{2} \boldsymbol{u}(n-1) \boldsymbol{u}^{T}(n-1) \boldsymbol{p}^{o} \boldsymbol{p}^{o T} \boldsymbol{u}(n-1) \boldsymbol{u}^{T}(n-1) \\
& +\rho^{2} u(n) \boldsymbol{u}(n-1) \boldsymbol{u}^{T}(n-1) \boldsymbol{p}^{o} \boldsymbol{u}^{T}(n-1) \\
& -\rho u(n) \boldsymbol{u}(n-1) \boldsymbol{v}_{p}^{T}(n)+\rho^{2} u^{2}(n) \boldsymbol{u}(n-1) \boldsymbol{u}^{T}(n-1) \\
& +\rho^{2} u(n) \boldsymbol{u}(n-1) \boldsymbol{v}_{p}^{T}(n) \boldsymbol{u}(n-1) \boldsymbol{u}^{T}(n-1) \\
& +\rho^{2} u(n) \boldsymbol{u}(n-1) \boldsymbol{p}^{o T} \boldsymbol{u}(n-1) \boldsymbol{u}^{T}(n-1)
\end{aligned}
$$

Taking the expected value of (11) and making the same approximations as in (7) yields

$$
\begin{aligned}
& \boldsymbol{K}_{p}(n+1)=\boldsymbol{K}_{p}(n)-\rho\left[\boldsymbol{K}_{p}(n) \boldsymbol{R}_{u u}(0)+\boldsymbol{R}_{u u}(0) \boldsymbol{K}_{p}(n)\right. \\
& +\mathrm{E}\left[\boldsymbol{v}_{p}(n)\right] \boldsymbol{p}^{o T} \boldsymbol{R}_{u u}(0)+\mathrm{E}\left[\boldsymbol{v}_{p}(n)\right] \boldsymbol{r}_{u}^{T}(1)+\boldsymbol{r}_{u}(1) \mathrm{E}\left[\boldsymbol{v}_{p}^{T}(n)\right] \\
& +\boldsymbol{R}_{u u}(0) \boldsymbol{p}^{o} \mathrm{E}\left[\boldsymbol{v}_{p}^{T}(n)\right]+\rho^{2}\left[\boldsymbol{R}_{u u}(0) \operatorname{tr}\left(\boldsymbol{R}_{u u}(0) \boldsymbol{K}_{p}(n)\right)\right. \\
& +2 \boldsymbol{R}_{u u}(0) \boldsymbol{K}_{p}(n) \boldsymbol{R}_{u u}(0)+\boldsymbol{R}_{u u}(0) \operatorname{tr}\left(\boldsymbol{R}_{u u}(0) \mathrm{E}\left[\boldsymbol{v}_{p}(n)\right] \boldsymbol{p}^{o T}\right) \\
& +2 \mathrm{E}\left[\boldsymbol{v}_{p}(n)\right] \boldsymbol{p}^{o T} \boldsymbol{R}_{u u}(0)+\boldsymbol{R}_{u u}(0) \operatorname{tr}\left(\boldsymbol{R}_{u u}(0) \boldsymbol{p}^{o} \boldsymbol{p}^{o T}\right) \\
& +2 \boldsymbol{R}_{u u}(0) \boldsymbol{p}^{o} \boldsymbol{p}^{o T} \boldsymbol{R}_{u u}(0)+\boldsymbol{R}_{u u}(0) \operatorname{tr}\left(\boldsymbol{R}_{u u}(0) \boldsymbol{p}^{o} \mathrm{E}\left[\boldsymbol{v}_{p}^{T}(n)\right]\right) \\
& \left.+2 \boldsymbol{p}^{o} \mathrm{E}\left[\boldsymbol{v}_{p}^{T}(n)\right] \boldsymbol{R}_{u u}(0)\right]+\rho^{2}\left[\boldsymbol{R}_{u u}(0) \mathrm{E}\left[\boldsymbol{v}_{p}(n)\right] \boldsymbol{r}_{u}^{T}(1)\right. \\
& +\boldsymbol{r}_{u}(1) \mathrm{E}\left[\boldsymbol{v}_{p}^{T}(n)\right] \boldsymbol{R}_{u u}(0)+\boldsymbol{R}_{u u}(0) \operatorname{tr}\left(\boldsymbol{r}_{u}(1) \mathrm{E}\left[\boldsymbol{v}_{p}^{T}(n)\right]\right) \\
& +\boldsymbol{R}_{u u}(0) \boldsymbol{p}^{o} \boldsymbol{r}_{u}^{T}(1)+\boldsymbol{r}_{u}(1) \boldsymbol{p}^{o T} \boldsymbol{R}_{u u}(0)+\boldsymbol{R}_{u u}(0) \operatorname{tr}\left(\boldsymbol{r}_{u}(1) \boldsymbol{p}^{o T}\right) \\
& +\boldsymbol{r}_{u}(1) \mathrm{E}\left[\boldsymbol{v}_{q}^{T}(n)\right] \boldsymbol{R}_{u u}(0)+\boldsymbol{R}_{u u}(0) \mathrm{E}\left[\boldsymbol{v}_{p}(n)\right] \boldsymbol{r}_{u}^{T}(1) \\
& +\boldsymbol{R}_{u u}(0) \operatorname{tr}\left(\mathrm{E}\left[\boldsymbol{v}_{p}(n)\right] \boldsymbol{r}_{u}^{T}(1)\right)+\boldsymbol{r}_{u}(1) \boldsymbol{p}^{o T} \boldsymbol{R}_{u u}(0) \\
& +\boldsymbol{R}_{u u}(0) \boldsymbol{p}^{o} \boldsymbol{r}_{u}^{T}(1)+\boldsymbol{R}_{u u}(0) \operatorname{tr}\left(\boldsymbol{p}^{o} \boldsymbol{r}_{u}^{T}(1)\right) \\
& \left.+2 \boldsymbol{r}_{u}(1) \boldsymbol{r}_{u}^{T}(1)+\boldsymbol{R}_{u u}(0) r_{u}(0)\right]
\end{aligned}
$$

An expression for $r_{u}(l)$ is determined in the Appendix, Eq. (34), assuming an AR model for $x(n)$. Expressions for $\mathrm{E}\{u(n) \boldsymbol{u}(n-$ 1) $\left.\boldsymbol{u}^{T}(n-1) \boldsymbol{v}_{p}(n) \boldsymbol{u}^{T}(n-1)\right\}, \mathrm{E}\left\{u(n) \boldsymbol{u}(n-1) \boldsymbol{v}_{p}^{T}(n) \boldsymbol{u}(n-\right.$ 1) $\left.\boldsymbol{u}^{T}(n-1)\right\}$ and $\mathrm{E}\left\{u^{2}(n) \boldsymbol{u}(n-1) \boldsymbol{u}^{T}(n-1)\right\}$ are obtained in sections V-B, V-C and V-D of the Appendix, respectively. The remaining expectations in (12) were derived as in [16].

Eq. (12) can be written as

$$
\begin{aligned}
\boldsymbol{K}_{p}(n+1)= & \left(\boldsymbol{I}-\rho \boldsymbol{R}_{u u}(0)\right) \boldsymbol{K}_{p}(n)\left(\boldsymbol{I}-\rho \boldsymbol{R}_{u u}(0)\right) \\
& +\rho^{2} \boldsymbol{R}_{u u}(0) \boldsymbol{K}_{p}(n) \boldsymbol{R}_{u u}(0) \\
& +\rho^{2} \boldsymbol{R}_{u u}(0) \operatorname{tr}\left(\boldsymbol{R}_{u u}(0) \boldsymbol{K}_{p}(n)\right)+\boldsymbol{C}_{q}(n)
\end{aligned}
$$

where $I$ is the $M \times M$ identity matrix and

$$
\begin{aligned}
& \boldsymbol{C}_{q}(n)= \\
& -\rho\left[\mathrm{E}\left[\boldsymbol{v}_{p}(n)\right] \boldsymbol{p}^{o T} \boldsymbol{R}_{u u}(0)+\mathrm{E}\left[\boldsymbol{v}_{p}(n)\right] \boldsymbol{r}_{u}^{T}(1)+\boldsymbol{r}_{u}(1) \mathrm{E}\left[\boldsymbol{v}_{p}^{T}(n)\right]\right. \\
& \left.+\boldsymbol{R}_{u u}(0) \boldsymbol{p}^{o} \mathrm{E}\left[\boldsymbol{v}_{p}^{T}(n)\right]\right]+\rho^{2}\left[\boldsymbol{R}_{u u}(0) \operatorname{tr}\left(\boldsymbol{R}_{u u}(0) \boldsymbol{p}^{o} \boldsymbol{p}^{o T}\right)\right. \\
& +2 \boldsymbol{R}_{u u}(0) \boldsymbol{p}^{o} \boldsymbol{p}^{o T} \boldsymbol{R}_{u u}(0)+2 \boldsymbol{R}_{u u}(0) \boldsymbol{p}^{o} \boldsymbol{r}_{u}^{T}(1) \\
& +2 \boldsymbol{r}_{u}(1) \boldsymbol{p}^{o T} \boldsymbol{R}_{u u}(0)+\boldsymbol{R}_{u u}(0) \operatorname{tr}\left(\boldsymbol{r}_{u}(1) \boldsymbol{p}^{o T}\right) \\
& +\boldsymbol{R}_{u u}(0) \operatorname{tr}\left(\boldsymbol{p}^{o} \boldsymbol{r}_{u}^{T}(1)\right)+2 \boldsymbol{r}_{u}(1) \boldsymbol{r}_{u}^{T}(1)+\boldsymbol{R}_{u u}(0) r_{u}(0) \\
& +\boldsymbol{R}_{u u}(0) \operatorname{tr}\left(\boldsymbol{R}_{u u}(0) \mathrm{E}\left[\boldsymbol{v}_{p}(n)\right] \boldsymbol{p}^{o T}\right)+2 \mathrm{E}\left[\boldsymbol{v}_{p}(n)\right] \boldsymbol{p}^{o T} \boldsymbol{R}_{u u}(0) \\
& \left.+\boldsymbol{R}_{u u}(0) \operatorname{tr}\left(\boldsymbol{R}_{u u}(0) \boldsymbol{p}^{o} \mathrm{E}\left[\boldsymbol{v}_{p}^{T}(n)\right]\right)+2 \boldsymbol{p}^{o} \mathrm{E}\left[\boldsymbol{v}_{p}^{T}(n)\right] \boldsymbol{R}_{u u}(0)\right] \\
& +2 \boldsymbol{R}_{u u}(0) \mathrm{E}\left[\boldsymbol{v}_{p}(n)\right] \boldsymbol{r}_{u}^{T}(1)+2 \boldsymbol{r}_{u}(1) \mathrm{E}\left[\boldsymbol{v}_{p}^{T}(n)\right] \boldsymbol{R}_{u u}(0) \\
& \left.+\boldsymbol{R}_{u u}(0) \operatorname{tr}\left(\boldsymbol{r}_{u}(1) \mathrm{E}\left[\boldsymbol{v}_{p}^{T}(n)\right]\right)+\boldsymbol{R}_{u u}(0) \operatorname{tr}\left(\mathrm{E}\left[\boldsymbol{v}_{p}(n)\right] \boldsymbol{r}_{u}^{T}(1)\right)\right]
\end{aligned}
$$

To study the steady-state behavior of $\boldsymbol{K}_{p}(n)$ (and thus of $\left.\mathrm{E}\left\{x_{q}(n) \boldsymbol{u}_{q}(n)\right\}\right)$, we write $\boldsymbol{R}_{u u}(0)=\boldsymbol{Q} \boldsymbol{\Lambda} \boldsymbol{Q}^{T}$, where the columns of $\boldsymbol{Q}$ are the orthonormal eingenvectors of $\boldsymbol{R}_{u u}(0)$ and $\boldsymbol{\Lambda}$ is its diagonal eigenvalue matrix with elements $\lambda_{i}, i=1, \cdots, M$. We also define $\boldsymbol{H}(n)=\boldsymbol{Q}^{T} \boldsymbol{K}_{p}(n) \boldsymbol{Q}$ so that $\boldsymbol{K}_{p}(n)=\boldsymbol{Q} \boldsymbol{H}(n) \boldsymbol{Q}^{T}$. Then, (13) can be writen as

$$
\begin{aligned}
\boldsymbol{Q H}(n+1) \boldsymbol{Q}^{T} & =\left(\boldsymbol{I}-\rho \boldsymbol{Q} \boldsymbol{\Lambda} \boldsymbol{Q}^{T}\right) \boldsymbol{Q} \boldsymbol{H}(n) \boldsymbol{Q}^{T}\left(\boldsymbol{I}-\rho \boldsymbol{Q} \boldsymbol{\Lambda} \boldsymbol{Q}^{T}\right) \\
& +\rho^{2} \boldsymbol{Q} \boldsymbol{\Lambda} \boldsymbol{Q}^{T} \boldsymbol{Q} \boldsymbol{H}(n) \boldsymbol{Q}^{T} \boldsymbol{Q} \boldsymbol{\Lambda} \boldsymbol{Q}^{T} \\
& +\rho^{2} \boldsymbol{Q} \boldsymbol{\Lambda} \boldsymbol{Q}^{T} \operatorname{tr}\left(\boldsymbol{Q} \boldsymbol{\Lambda} \boldsymbol{Q}^{T} \boldsymbol{Q} \boldsymbol{H}(n) \boldsymbol{Q}^{T}\right)+\boldsymbol{C}_{q}(n)
\end{aligned}
$$

Using $\boldsymbol{Q}^{T} \boldsymbol{Q}=\boldsymbol{I}$ and pre- and post-multiplying (15) by $\boldsymbol{Q}^{T}$ and $\boldsymbol{Q}$ respectively yields:

$$
\boldsymbol{H}(n+1)=\boldsymbol{F} \boldsymbol{H}(n) \boldsymbol{F}+\rho^{2} \boldsymbol{\Lambda} \boldsymbol{H}(n) \boldsymbol{\Lambda}+\rho^{2} \boldsymbol{\Lambda} t r(\boldsymbol{\Lambda} \boldsymbol{H}(n))+\boldsymbol{C}_{q}^{\prime}
$$

where $\boldsymbol{C}_{q}^{\prime}=\boldsymbol{Q}^{T} \boldsymbol{C}_{q}(n) \boldsymbol{Q}$ and $\boldsymbol{F}=(\boldsymbol{I}-\rho \boldsymbol{\Lambda}) . \boldsymbol{F}$ is a diagonal matrix with diagonal element vector $\boldsymbol{f}=\left[f_{1}, \cdots f_{M}\right]^{T}$, where $f_{i}=1-\rho \lambda_{i}$.

Elements $h_{i j}$ of (16) for $i \neq j$ are given by

$$
h_{i j}(n+1)=f_{i} f_{j} h_{i j}(n)+\rho^{2} \lambda_{i} \lambda_{j} h_{i j}(n)+c_{i j}^{\prime}
$$

Thus, (17) for $n \rightarrow \infty$ is given by:

$$
\lim _{n \rightarrow \infty} h_{i j}(n)=\frac{c_{i j}^{\prime}}{\rho\left(\lambda_{i}+\lambda_{j}-2 \rho \lambda_{i} \lambda_{j}\right)} \quad \text { for } i \neq j
$$

where $c_{i j}^{\prime}$ are elements of the matrix $C_{q}^{\prime}$.

Denoting for simplicity $h_{i}(n)$ the diagonal elements $h_{i i}(n)$ of $\boldsymbol{H}(n),(16)$ yields

$$
h_{i}(n+1)=f_{i}^{2} h_{i}(n)+\rho^{2} \lambda_{i}^{2} h_{i}(n)+\rho^{2} \lambda_{i} \boldsymbol{\lambda}^{T} \boldsymbol{h}(n)+c_{i i}^{\prime}
$$

where $\boldsymbol{\lambda}=\left[\lambda_{1}, \ldots, \lambda_{M}\right]^{T}$ and $\boldsymbol{h}(n)=\left[h_{1}(n), \ldots, h_{M}(n)\right]^{T}$. Thus, we can write,

$$
\boldsymbol{h}(n+1)=\boldsymbol{B} \boldsymbol{h}(n)+\boldsymbol{c}^{\prime}
$$

where $c^{\prime}$ is a vector that contains the diagonal elements of $C_{q}^{\prime}$ and $\boldsymbol{B}$ is an $M \times M$ matrix with elements

$$
b_{i j}=\left\{\begin{array}{lll}
f_{i}^{2}+2 \rho^{2} \lambda_{i}^{2}, & \text { for } & i=j \\
\rho^{2} \lambda_{i} \lambda_{j}, & \text { for } & i \neq j
\end{array}\right.
$$

The steady-state solution of (20) is given by:

$$
\boldsymbol{h}(\infty)=[\boldsymbol{I}-\boldsymbol{B}]^{-1} \boldsymbol{c}^{\prime}
$$


Denoting $\boldsymbol{B}^{\prime}=[\boldsymbol{I}-\boldsymbol{B}]^{-1}$, we have:

$$
h_{i}(\infty)=\sum_{k=1}^{M} b_{i k}^{\prime} c_{k}^{\prime}
$$

Summarizing, the elements of $\boldsymbol{H}(\infty)$ are given by

$$
h_{i j}(\infty)=\left\{\begin{array}{lll}
\frac{c_{i j}^{\prime}}{\rho\left(\lambda_{i}+\lambda_{j}-2 \rho \lambda_{i} \lambda_{j}\right)}, & \text { for } & i \neq j \\
\sum_{k=1}^{M} b_{i k}^{\prime} c_{k}^{\prime}, & \text { for } & i=j
\end{array}\right.
$$

where $\boldsymbol{K}_{p}(\infty)=\boldsymbol{Q} \boldsymbol{H}(\infty) \boldsymbol{Q}^{T}$.

Equation (23) shows how $\boldsymbol{K}_{p}(\infty)$ depends on the size of $\rho$ and on the eigenvalues de $\boldsymbol{R}_{u u}(0)$. Note also that these eigenvalues are functions of the delay $D$ through $r_{u}(l)$ (see (34)).

The model for the bias (4) still requires a mathematical expression for $\boldsymbol{R}_{u_{q} u_{q}}(n)$. This expression is derived next.

\section{Correlation Matrix $\boldsymbol{R}_{u_{q} u_{q}}(n)$}

The elements of $\boldsymbol{R}_{u_{q} u_{q}}(n)$ are given by

$$
\left[\boldsymbol{R}_{u_{q} u_{q}}(n)\right]_{i j}=\mathrm{E}\left\{u_{q}(n-i) u_{q}(n-j)\right\} .
$$

Since $u_{q}(n-i)=u(n)+\boldsymbol{p}^{T}(n) \boldsymbol{u}(n-i-1)$ we have

$$
\begin{aligned}
{\left[\boldsymbol{R}_{u_{q} u_{q}}(n)\right]_{i j}=} & \mathrm{E}\left\{\left[u(n-i)+\boldsymbol{p}^{T}(n) \boldsymbol{u}(n-i-1)\right]\right. \\
& \left.\times\left[u(n-j)+\boldsymbol{p}^{T}(n) \boldsymbol{u}(n-j-1)\right]\right\}
\end{aligned}
$$

Assuming slow convergence as done in section III-A such that $\boldsymbol{p}(n)=\boldsymbol{p}(n-1)=\boldsymbol{p}(n-N)$ and using $\boldsymbol{p}(n)=\boldsymbol{v}_{p}(n)+\boldsymbol{p}^{o}$ in (24) yields a recursion fo $\boldsymbol{R}_{u_{q} u_{q}}(n)$ as a function ot time.

$$
\begin{aligned}
& {\left[\boldsymbol{R}_{u_{q} u_{q}}(n)\right]_{i j}=} \\
& \mathrm{E}\left\{u(n-i) u(n-j)+u(n-i) \boldsymbol{v}_{p}^{T}(n) \boldsymbol{u}(n-j-1)\right. \\
& +u(n-i) \boldsymbol{p}^{o T} \boldsymbol{u}(n-j-1)+\boldsymbol{v}_{p}^{T}(n) \boldsymbol{u}(n-i-1) u(n-j) \\
& +\boldsymbol{v}_{p}^{T}(n) \boldsymbol{u}(n-i-1) \boldsymbol{u}^{T}(n-j-1) \boldsymbol{v}_{p}(n) \\
& +\boldsymbol{v}_{p}^{T}(n) \boldsymbol{u}(n-i-1) \boldsymbol{u}^{T}(n-j-1) \boldsymbol{p}^{o} \\
& +\boldsymbol{p}^{o T} \boldsymbol{u}(n-i-1) u(n-j) \\
& +\boldsymbol{p}^{o T} \boldsymbol{u}(n-i-1) \boldsymbol{u}^{T}(n-j-1) \boldsymbol{v}_{p}(n) \\
& \left.+\boldsymbol{p}^{o T} \boldsymbol{u}(n-i-1) \boldsymbol{u}^{T}(n-j-1) \boldsymbol{p}^{o}\right\}
\end{aligned}
$$

Taking the expected values,

$$
\begin{aligned}
& {\left[\boldsymbol{R}_{u_{q} u_{q}}(n)\right]_{i j}=} \\
& r_{u}(j-i)+\boldsymbol{p}^{o T} \boldsymbol{r}_{u}(j+1-i)+\boldsymbol{p}^{o T} \boldsymbol{r}_{u}(j-(i+1)) \\
& +\operatorname{tr}\left\{\boldsymbol{R}_{u}(j-i) \boldsymbol{K}_{p}(n)\right\}+\operatorname{tr}\left\{\boldsymbol{R}_{u}(j-i) \boldsymbol{p}^{o} \boldsymbol{p}^{o T}\right\} \\
& +\mathrm{E}\left[\boldsymbol{v}_{p}(n)\right]^{T} \boldsymbol{r}_{u}(j+1-i)+\mathrm{E}\left[\boldsymbol{v}_{p}(n)\right]^{T} \boldsymbol{r}_{u}(j-(i+1)) \\
& +\operatorname{tr}\left\{\boldsymbol{R}_{u}(j-i) \boldsymbol{p}^{o} \mathrm{E}\left[\boldsymbol{v}_{p}(n)\right]^{T}\right\}+\operatorname{tr}\left\{\boldsymbol{R}_{u}(j-i) \mathrm{E}\left[\boldsymbol{v}_{p}(n)\right] \boldsymbol{p}^{o T}\right\}
\end{aligned}
$$

Using (26) with (8) and the expression derived in the Appendix for $r_{u}(l)$ yields an analytical model for $\boldsymbol{R}_{u_{q} u_{q}}(n)$. Then (4) can be resolved through equations (7), (23) and (26), proving the desired transient model for the bias. In steady-state, $\boldsymbol{R}_{u_{q} u_{q}}(\infty)$ can be obtained from (26) using $\boldsymbol{K}_{p}(\infty)$ obtained in the previous section and

$$
E\left[\boldsymbol{v}_{p}(\infty)\right]=-\boldsymbol{R}_{u u}(0)^{-1} \boldsymbol{r}_{u}(1)-\boldsymbol{p}^{o}
$$

determined from (8) as $n \rightarrow \infty$.

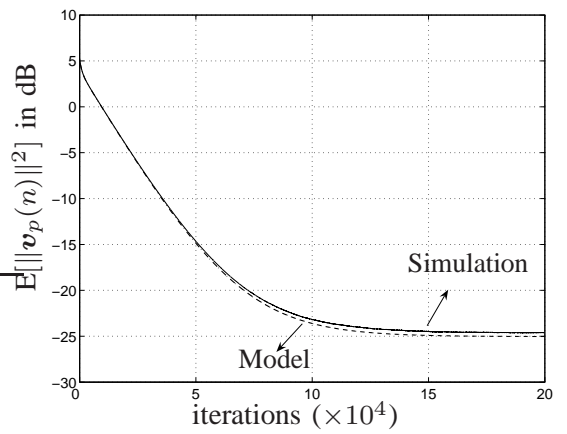

Fig. 3. Predictor MSD for the structure in Fig. 2.

\section{Simulation Results}

This section presents Monte Carlo (MC) simulations to verify the theoretical model derived. The structure of Fig. 2 was utilized and the parameters used were $\mu=0.001, \rho=0.0001, D=15, \sigma_{x}^{2}=1$ (using $\left.\sigma_{\eta}^{2}=0.1875\right), \sigma_{\zeta}^{2}=10^{-5}$ and $H(z)=1 /\left(1-1.5 z^{-1}+z^{-2}-\right.$ $0.25 z^{-3}$ ) [17] (thus $\boldsymbol{p}^{\mathrm{O}}=[-1.5 ; 1 ;-0.25]^{T}$ ). The feedback path was given by $\boldsymbol{w}^{o}=[-0.0016,0.0016,0.0046,0.0502,-0.0691]^{T}$ (first 5 samples of an actual feedback path response). MC simulations are averaged over 200 realizations.

Fig. 3 shows theoretical (Eq. (13)) and MC simulation results for the mean-square deviation (MSD) $\mathrm{E}\left[\left\|\boldsymbol{v}_{p}(n)\right\|^{2}\right]=\sum_{i=1}^{M}\left[\boldsymbol{K}_{p}(n)\right]_{i i}$. Excellent matching can be verified in both the transient and steadystate phases. Fig. 4 shows the theoretical MSD for $\mu=0.001$ and for differents values of both the delay $D$ and PEF step size $\rho$. Other parameters were the same as in Fig. 3. These results show that larger feedforward delays lead to faster convergence and lower MSD for a fixed step size. Also, as expected, reducing the step size $\rho$ leads to slower convergence and lower steady-state MSD.

Fig. 5 shows the MC simulation result and the theoretically predicted steady-state value of the bias vector norm for $D=2$. Table I shows the steady-state weight bias coefficients evaluated from (4) using MC simulations and using the theoretical model provided by (13), (7), (23), (26) and (4). Excellent agreement can again be verified.

TABLE I

THEORETICAL AND SIMULATED STEADY-STATE WEIGHT BIAS.

\begin{tabular}{c|ccccc} 
& $\hat{w}_{0}(\infty)$ & $\hat{w}_{1}(\infty)$ & $\hat{w}_{2}(\infty)$ & $\hat{w}_{3}(\infty)$ & $\hat{w}_{4}(\infty)$ \\
\hline Theory & -0.0023 & -0.0099 & -0.0089 & -0.0042 & 0 \\
Simulation & 0.0005 & -0.00103 & -0.0090 & -0.0044 & 0.0001
\end{tabular}

Fig. 6 shows the variation of the signal-to-noise ratio in $\hat{y}(n)$ where $\boldsymbol{w}^{o T} \boldsymbol{u}(n)$ is the desirable signal and $\mathcal{B}^{T} \boldsymbol{u}(n)$ is the noise. Thus, SNR $=10 \log \left\{\left\|\boldsymbol{w}^{o}\right\|^{2} /\|\mathcal{B}\|^{2}\right\}$ is evaluated using the proposed model as a function of the predictor step size $\rho$ and delay $D$. The remaining parameters are the same listed at the first paragraph of this section.

\section{CONCLUSiOnS}

This paper presented a statistical analysis for bias resulting from a well known structure for acoustic feedback cancellation is hearing aids. The structure has two adaptive systems, one estimator and one predictor. An analytical model has been derived for the behavior of the bias during the transient and steady-state phases of adaptation for the LMS algorithm applied to both the estimator and the predictor. The new model accurately predicts the variations of the bias as a function of the feedforward path delay and of the adaptive predictor 


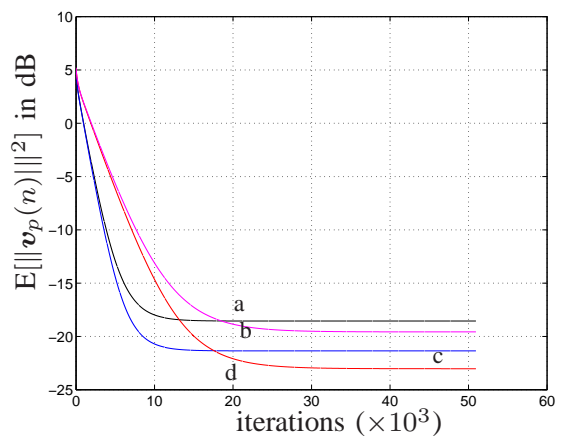

Fig. 4. Predictor MSD as a function of $D$ and $\rho$ : a) $D=2 ; \rho=0.001$, b) $D=2 ; \rho=0.0005$, c) $D=15 ; \rho=0.001$, d) $D=15 ; \rho=0.0005$.

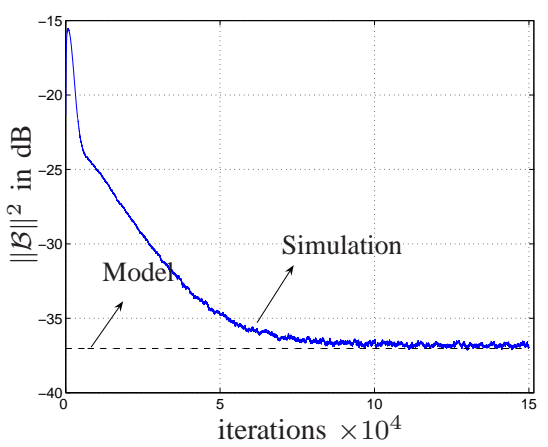

Fig. 5. Norm of the bias vector $(\mathrm{dB})$ for $D=2$

step size. The theoretical predictions show excellent agreement with Monte Carlo simulations. It is expected that the new results be useful in the design of feedback cancellation systems for hearing aids.

\section{ACKNOWLEDGMENTS}

This work has been partially supported CNPq under grants Nos. 307024/2006-7 and 470792/2006-0, by CAPES and by the University of Antofagasta-Chile.

\section{APPENDIX}

A. Evaluation of $r_{u}(l)$ and $r_{x u}(l)$

This section determines the correlations $r_{u}(l)$ and $r_{x u}(l)$ from the stationary closed loop subsystem in the top part of Fig. 2 for

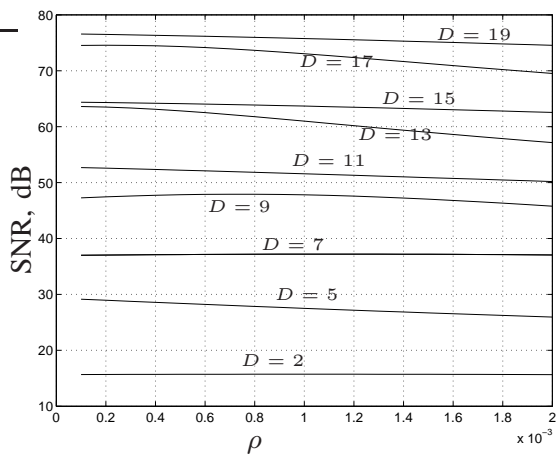

Fig. 6. Signal-to-noise ratio $\mathrm{SNR}=10 \log \left(\left\|\boldsymbol{w}^{o}\right\|^{2} /\|\mathcal{B}\|^{2}\right)$ as a function of forward path delay $D$ and predictor step size $\rho$. an autoregressive input $x(n)$. Since $\eta(n)$ and $\zeta(n)$ are assumed Gaussian, both $x(n)$ and $u(n)$ are Gaussian processes.

1) Autocorrelation $r_{u}(l)$ : Defining the time-invariant weight error vector $\boldsymbol{v}^{c}=\boldsymbol{w}^{c}-\boldsymbol{w}^{o}$, we have

$$
u(n)=\gamma(n)-G \boldsymbol{u}^{T}(n-D) \boldsymbol{v}^{c}
$$

where $\gamma(n)=\zeta(n)+G x(n-D)$. Taking the z-transform of (28) yields

$$
H_{u}(z)=\frac{U(z)}{\Gamma(z)}=\frac{1}{1+G \boldsymbol{\psi}\left(z^{-1}\right) \boldsymbol{v}^{c} z^{-D}}
$$

with $\psi\left(z^{-1}\right)=\left[1, z^{-1}, z^{-2}, \cdots, z^{-N+1}\right]^{T}$. Since $\zeta(n)$ is independent of $x(n)$,

$$
\begin{gathered}
R_{u}(z)=\frac{R_{\gamma u}(z)}{1+G \boldsymbol{\psi}\left(z^{-1}\right) \boldsymbol{v}^{c} z^{-D}} \\
R_{\gamma u}(z)=\frac{R_{\zeta}(z)}{1+G \boldsymbol{\psi}(z) \boldsymbol{v}^{c} z^{D}}+\frac{G^{2} R_{x}(z)}{1+G \boldsymbol{\psi}(z) \boldsymbol{v}^{c} z^{D}}
\end{gathered}
$$

where $R_{u}(z)$ is the complex spectral density of $u(n)$ and $R_{\gamma u}(z)$ is the complex cross-spectral density of $u(n)$ and $\gamma(n)$.

For $x(n)$ autoregressive, $R_{x}(z)=H(z) H^{*}\left(1 / z^{*}\right) \sigma_{\eta}^{2}$ with

$$
H(z)=\frac{1}{\prod_{i=1}^{M}\left(1+a_{i} z^{-1}\right)} .
$$

Assuming stability of (29) and applying the Residue Theorem to (31), straightforward calculation leads to

$$
\begin{aligned}
r_{\gamma u}(l)= & \sigma_{\zeta}^{2} \delta(l)+\sum_{k=1}^{M} \frac{G^{2} \sigma_{\eta}^{2}\left(-a_{k}\right)^{M}\left(-a_{k}\right)^{l-1}}{\prod_{\substack{i=1 \\
k \neq i}}^{M}\left(a_{i}-a_{k}\right) \prod_{j=1}^{M}\left(1-a_{j} a_{k}\right)} \\
& \times \frac{1}{\left(1+G \boldsymbol{\psi}\left(-a_{k}\right) \boldsymbol{v}^{c}\left(-a_{k}\right)^{D}\right)}, \quad l \geq 0 .
\end{aligned}
$$

Finally, it follows from (30) that

$$
r_{u}(l)=r_{\gamma u}(l)-G \boldsymbol{r}_{u}^{T}(l-D) \boldsymbol{v}^{c}
$$

where $\boldsymbol{r}_{u}(l-D)=\left[r_{u}(l-D), \ldots, r_{u}(l-D-N+1)\right]^{T}$.

2) Cross-correlation $r_{x u}(l)$ : We have that $r_{x u}(l)=\mathrm{E}\{x(n+$ l) $u(n)\}=G h(-l) * r_{x}(l+D)$ where $h(n)$ is the inverse z-transform of (32) and $*$ means linear convolution. Thus,

$$
R_{x u}(z)=G H^{*}\left(1 / z^{*}\right) R_{x}(z) z^{D} .
$$

Substituting (32) and $R_{x}(z)=H(z) H^{*}\left(1 / z^{*}\right) \sigma_{\eta}^{2}$ in (35) and using the Residue Theorem yields

$$
\begin{aligned}
r_{x u}(l)= & \sum_{k=1}^{M} \frac{G \sigma_{\eta}^{2}\left(-a_{k}\right)^{D+M}\left(-a_{k}\right)^{l-1}}{\prod_{\substack{i=1 \\
k \neq i}}^{M}\left(a_{i}-a_{k}\right) \prod_{j=1}^{M}\left(1-a_{j} a_{k}\right)} \\
& \times \frac{1}{\left(1+G \boldsymbol{\psi}\left(-a_{k}\right) \boldsymbol{v}^{c}\left(-a_{k}\right)^{D}\right)}, \quad l \geq 0
\end{aligned}
$$

and

$$
\begin{aligned}
r_{x u}(l)= & \sum_{k=1}^{M} \frac{G \sigma_{\eta}^{2}\left(-a_{k}\right)^{D+M}}{\left(-a_{k}\right)^{l+1} \prod_{\substack{i=1 \\
k \neq i}}^{M}\left(a_{i}-a_{k}\right) \prod_{j=1}^{M}\left(1-a_{j} a_{k}\right)} \\
& \times \frac{1}{\left(1+G \psi\left(-a_{k}\right) \boldsymbol{v}^{c}\left(-a_{k}\right)^{D}\right)}, \quad l<0 .
\end{aligned}
$$


B. $E\left\{u(n) \boldsymbol{u}(n-1) \boldsymbol{u}^{T}(n-1) \boldsymbol{v}_{p}(n) \boldsymbol{u}^{T}(n-1)\right\}$

Define $M_{r p}=\sum_{s=1}^{M} \mathrm{E}\left[u(n) u(n-r) u(n-s) v_{p}(n-s+1) u(n-\right.$ $p)$ ]. Taking the expectation of $M_{r p}$ conditioned on $v_{p}(n-s+1)$ and neglecting the statistical dependence between $\boldsymbol{u}(n-l)$ and $\boldsymbol{v}_{p}(n-k)$ for all $l$ and $k$ leads to

$$
\begin{aligned}
\mathrm{E}\left[\left.M_{r p}\right|_{v_{p}(n-s+1)}\right] & =\sum_{s=1}^{M} \mathrm{E}[u(n) u(n-r) u(n-s) u(n-p)] \\
& \times v_{p}(n-s+1)
\end{aligned}
$$

where $r=1, \cdots, M$ and $p=1, \cdots, M$. Assuming the variables in the expectation to be jointly Gaussian,

$$
\begin{aligned}
\mathrm{E} & {\left[\mathbf{M}_{r p}\right]=\left\{\sum_{s=1}^{M} \mathrm{E}[u(n) \boldsymbol{u}(n-1)]_{r, 1} \mathrm{E}\left[\boldsymbol{u}(n-1) \boldsymbol{u}^{T}(n-1)\right]_{s, p}\right.} \\
& +\sum_{s=1}^{M} \mathrm{E}[u(n) \boldsymbol{u}(n-1)]_{s, 1} \mathrm{E}[\boldsymbol{u}(n-1) \boldsymbol{u}(n-1)]_{r p} \\
& \left.+\sum_{s=1}^{M} \mathrm{E}[u(n) \boldsymbol{u}(n-1)]_{p, 1} \mathrm{E}\left[\boldsymbol{u}(n-1) \boldsymbol{u}^{T}(n-1)\right]_{r, s}\right\} \boldsymbol{v}_{p}^{T}(n)_{s, 1}
\end{aligned}
$$

which leads to

$$
\begin{aligned}
\mathrm{E}\left[\mathrm{M}_{r p}\right] & =\boldsymbol{r}_{u}(1) \mathrm{E}\left[\boldsymbol{v}_{p}^{T}(n)\right] \boldsymbol{R}_{u u}(0)+\boldsymbol{R}_{u u}(0) \operatorname{tr}\left(\boldsymbol{r}_{u}(1) \mathrm{E}\left[\boldsymbol{v}_{p}^{T}(n)\right]\right) \\
& +\boldsymbol{R}_{u u}(0) \mathrm{E}\left[\boldsymbol{v}_{p}(n)\right] \boldsymbol{r}_{u}^{T}(1) .
\end{aligned}
$$

\section{C. $E\left\{u(n) \boldsymbol{u}(n-1) \boldsymbol{v}_{p}^{T}(n) \boldsymbol{u}(n-1) \boldsymbol{u}^{T}(n-1)\right\}$}

Define now $\left[M_{r p}\right]=\sum_{s=1}^{M} u(n) u(n-r) v_{p}(n-s) u(n-s) u(n-$ $p)$. Taking the expectation of $\left[M_{r p}\right]$ conditioned on $v_{p}(n-s)$ and neglecting the statistical dependence between $\boldsymbol{u}(n-l)$ and $\boldsymbol{v}_{p}(n-k)$ for all $l$ and $k$ we have,

$$
\mathrm{E}\left[M_{r p}\right]=\sum_{s=1}^{M} \mathrm{E}[u(n) u(n-r) u(n-s) u(n-p)] v_{p}(n-s)
$$

where $r=1, \cdots, M$ and $p=1, \cdots, M$. Assuming the variables in the expectation to be jointly Gaussian,

$$
\begin{aligned}
\mathrm{E} & {\left[\mathbf{M}_{r p}\right]=\left\{\sum_{s=1}^{M} \mathrm{E}[u(n) \boldsymbol{u}(n-1)]_{r, 1} \mathrm{E}\left[\boldsymbol{u}(n-1) \boldsymbol{u}^{T}(n-1)\right]_{s, p}\right.} \\
& +\sum_{s=1}^{M} \mathrm{E}[u(n) \boldsymbol{u}(n-1)]_{s, 1} \mathrm{E}\left[\boldsymbol{u}(n-1) \boldsymbol{u}^{T}(n-1)\right]_{r, p} \\
& \left.+\sum_{s=1}^{M} \mathrm{E}[u(n) \boldsymbol{u}(n-1)]_{p, 1} \mathrm{E}\left[\boldsymbol{u}(n-1) \boldsymbol{u}^{T}(n-1)\right]_{r, s}\right\} \boldsymbol{v}_{p}(n)_{s}, 1
\end{aligned}
$$

Thus,

$$
\begin{aligned}
\mathrm{E}\left[\mathbf{M}_{r p}\right] & =\boldsymbol{r}_{u}(1) \mathrm{E}\left[\boldsymbol{v}_{p}^{T}(n)\right] \boldsymbol{R}_{u u}(0)+\boldsymbol{R}_{u u}(0) \mathrm{E}\left[\boldsymbol{v}_{p}(n)\right] \boldsymbol{r}_{u}^{T}(1) \\
& +\boldsymbol{R}_{u u}(0) \operatorname{tr}\left(\boldsymbol{r}_{u}(1) \mathrm{E}\left[\boldsymbol{v}_{p}^{T}(n)\right]\right)
\end{aligned}
$$

\section{D. $E\left\{u^{2}(n) \boldsymbol{u}(n-1) \boldsymbol{u}^{T}(n-1)\right\}$}

Define now $\left[M_{r p}\right]=\sum_{s=1}^{M}=u(n-r) u(n) u(n) u(n-p)$ Taking the expectation of $\left[M_{r p}\right]$ yields

$$
\mathrm{E}\left[M_{r p}\right]=\sum_{s=1}^{M} \mathrm{E}[u(n-r) u(n) u(n) u(n-p)]
$$

where $r=1, \cdots, M$ and $p=1, \cdots, M$. Assuming all variables to be jointly Gaussian,

$$
\begin{aligned}
\mathrm{E}\left[\mathbf{M}_{r p}\right] & =\sum_{s=1}^{M} \mathrm{E}[\boldsymbol{u}(n-1) u(n)]_{r, 1} \mathrm{E}\left[u(n) \boldsymbol{u}^{T}(n-1)\right]_{p, 1} \\
& +\sum_{s=1}^{M} \mathrm{E}[\boldsymbol{u}(n-1) u(n)]_{r, 1} \mathrm{E}\left[u(n) \boldsymbol{u}^{T}(n-1)\right]_{p, 1} \\
& +\sum_{s=1}^{M} \mathrm{E}\left[\boldsymbol{u}(n-1) \boldsymbol{u}^{T}(n-1)\right]_{r, p} \mathrm{E}\left[u^{2}(n)\right] \\
& =2 \boldsymbol{r}_{u}(1) \boldsymbol{r}_{u}^{T}(1)+\boldsymbol{R}_{u u}(0) r_{u}(0) .
\end{aligned}
$$

\section{REFERENCIAS}

[1] K. Chung, "Challenges and recent develpoments in heraing aids part.1 speech undestanding in noise, microphone technologies and noise reduction algorithms," Trens In Amplification, vol. 8, no. 3, pp. 83-124, 2004.

[2] J. Hellgren, T.Lunner, and S. Arlinger, "Variations in the feedback of hearing aids," Acoustic Society of America, vol. 106, no. 5, pp. 28212833, November 1999.

[3] B. Rafaely, M. Roccasalva-Firenze, , and E. Payne, "Feedback path variability modeling for robust hearing aids," Acoustic Society of America, vol. 107, no. 5, pp. 2665-2673, May 2000.

[4] H. Joson, F. Asano, Y. Suzuki, and S. Toshio, "Adaptive feedback cancellation with frequency compresion for hearing aids," J. Acoust. Society of America, vol. 94, no. 6, pp. 3248-3254, December 1993.

[5] M. G. Siqueira and A. Alwan, "Steady-state analysis of continuous adaptation in acoustic feedback reduction systems for hearing-aids," IEEE Trans. on speech and audio processing, vol. 8, no. 4, pp. 443-453, July 2000.

[6] J. M. Kates, "Constrained adaptation for feedback cancellation in hearing aids," J. Acoust. Society of America, vol. 106, no. 2, pp. 1010-1019, August 1999.

[7] S.Gao, S.Soli, and H.-F. Chi, "Band-limited adaptive feedback canceller for hearing aids," European Patent W00 019 605, Sept., 1999.

[8] J. Benesty and Y. Huang, Adaptive Signal Processing : Applications to Real-World Problems. N.Y: Springer, 2003.

[9] L. Ljung, System identification, theory for the user. USA: Prentice Hall, 1987.

[10] J. Hellgren and U.Forssell, "Bias of feedback cancellation algorithms in hearing aids based on direct closed loop identification," IEEE Trans. Speech and audio processing, vol. 9, no. 7, pp. 906-913, November 2001.

[11] J. Hellgren, "Analysis of feedback cancellation in hearing aids with filtered-xlms and the direct method closed loop identification," IEEE Trans. Speech and audio processing, vol. 10, no. 2, pp. 119-131, February 2002.

[12] A. Spriet, I. Proudler, M. Moonen, and J. Wouters, "Adaptive feedback cancellation in hearing aids with linear prediction of the desired signal," IEEE Trans. on Signal processing, vol. 53, no. 10, pp. 3749-3763, October 2005.

[13] _ - "An instrumental variable method for adaptive feedback cancellation in hearing aids," in Proc. Int. Conf. Acoustic, Speech, Signal Processing, 2005, pp. 129-132.

[14] M. Mboup, M. Bonnet, and N. Bershad, "Lms coupled adaptive prediction and system identification: a statistical model and transient mean analysis," IEEE Transaccion Signal Processing, vol. 42, no. 10, pp. 2607-2615, October 1994.

[15] Y. Montenegro and J.C.M.Bermudez, "Mean weight behavior of coupled lms adaptive systems applied to acoustic feedback cancellation in hearing aids," in Int. Conf. Image and Signal Processing (ICISP), CherbourgOcteville, Normandy, France, 2008.

[16] S. Haykin, Adaptive Filter Theory 2nd ed. New Jersey: Prentice-Hall, 1991.

[17] A. Sugiyama, S. Ikeda, and A. Hirano, "A fast convergence algorithm for sparse-tap adaptive FIR filters identifying an unknown number of dispersive regions," IEEE Trans. Signal Process., vol. 50, no. 12, pp. 3008-3017, December 2002. 